\title{
A Gauge Theoretic Proof of the Abel-Jacobi Theorem
}

\author{
Gheorghe Ionesei
}

Abstract. We present a new, simple proof of the classical Abel-Jacobi theorem using some elementary gauge theoretic arguments.

\section{Introduction}

The classical Abel-Jacobi theorem is one of the most important results in the theory of Riemann surfaces. This theorem has a very interesting history [2]. The history starts with L. Euler's result that the functions defined by elliptic integrals

$$
\int \frac{d z}{\sqrt{P_{3}(z)}}
$$

where $P_{3}(z)$ is a degree 3 polynomial satisfy addition rules very similar to those involving the inverse trigonometric functions arising in integrals of the type

$$
\int \frac{d z}{\sqrt{P_{2}(z)}}, \quad \operatorname{deg} P_{2}=2
$$

The mathematics world was taken completely by surprise when a young Norwegian mathematician by the name of $\mathrm{N}$. Abel proved a very general addition rule for the most general class of algebraic integrals of the type

$$
\int \frac{P(z)}{w(z)} d z
$$

where $w(z)$ denotes an algebraic function implicitly defined by the polynomial equation $f(z, w)=0$. For $f(z, w)=w^{2}-P(z)$ one recovers the previously known formulae.

Jacobi later extended Abel's theorem to a complete description of all possible addition formulae. Abel's work was a remarkable tour de force at a time when none of the formalism we currently use to express and understand his result existed. It took more than a century for the modern formalism to develop and for mathematicians to get to the heart of the problem Abel solved. The introduction of Riemann surfaces and the recognition of the integral $(0.1)$ as the integral of a differential form resulted in the modern statement of Abel's theorem. The relevant Riemann surface is given by $f(z, w)=0$.

Received by the editors June 18, 1998; revised December 18, 1998 and August 3, 1999.

AMS subject classification: 58D27, 30F99.

Keywords: Abel-Jacobi theorem, abelian gauge theory.

(C) Canadian Mathematical Society 2000. 
At this point it is convenient to introduce the notion of divisor as a finite collection of points with integral multiplicities attached. The sum of the multiplicities is known as the degree of the divisor. The divisor of zeros and poles of a meromorphic function is called a principal divisor. The endpoints of paths of integration in Abel's theorem are given by a principal divisor: each goes from a pole to a zero. There are other problems with the integral (0.1). On one hand, the path of integration is unspecified but should avoid the points where $w=0$ and, on the other hand, the function $w$ is only defined locally; globally it is a multivalued function (think of the function $\sqrt{z}$ ). These integrals are only well defined modulo periods that can be organized as an Abelian (!) group.

Let us first observe that not all divisors are principal since the principal divisors have degree zero. Thus it is natural to restrict our attention to degree zero divisors. It was already known by the mathematicians of the time that not all degree zero divisors are principal!!! Abel's theorem can be interpreted as a necessary and sufficient condition for a divisor to be principal!

Consider a compact Riemann surface $\Sigma$. The space of divisors is the free abelian group $\operatorname{Div}(\Sigma)$, generated by the points of $\Sigma$. The degree is a group morphism

$$
\operatorname{deg}: \operatorname{Div}(\Sigma) \rightarrow \mathbb{Z}, \quad \operatorname{deg}\left(\sum n_{i}\left[p_{i}\right]\right)=\sum n_{i}
$$

Denote by $\operatorname{Div}_{0}(\Sigma)$ the kernel of the degree map which is the abelian group of degree zero divisors. Inside $\operatorname{Div}_{0}(\Sigma)$ sits the subgroup $\operatorname{PDiv}(\Sigma)$ consisting of divisors of meromorphic functions on $\Sigma$. Two questions arise naturally.

Q1 Which divisors are principal?

Q2 How large is the quotient group $\operatorname{Div}_{0}(\Sigma) / \operatorname{PDiv}(\Sigma)$ or, in other words, "how many" divisors are not principal?

Abel's theorem answers question Q1 while Jacobi's contribution is the answer to question Q2.

Let us describe their answers in modern terms. Fix a point $P \in \Sigma$. Then, for any divisor $D=\sum_{j=1}^{m} n_{j} P_{j} \in \operatorname{Div}_{0}(\Sigma)$, and any collection of paths $\mathbf{c}=\left(c_{j}\right)_{1 \leq j \leq m}$ connecting $P$ to $P_{j}$, we get a linear map

$$
\mathcal{P}_{\mathbf{c}}(D): H^{0}\left(K_{\Sigma}\right) \rightarrow \mathbb{C}, \quad \alpha \mapsto-2 \pi \sum_{j=1}^{m} n_{j} \int_{c_{j}} \alpha
$$

where $H^{0}\left(K_{\Sigma}\right)$ denotes the space of holomorphic $(1,0)$-forms on $\Sigma$. We regard $\mathcal{P}_{\mathbf{c}}(D)$ as an element in the complex dual $V:=H^{0}\left(K_{\Sigma}\right)^{*}$ of $H^{0}\left(K_{\Sigma}\right)$. The first homology group $H_{1}(\Sigma, \mathbb{Z})$ defines a lattice $\Lambda \subset V$ called the lattice of periods. To each cycle $c$ it associates the linear functional on $H^{0}\left(K_{\Sigma}\right)$ given by

$$
\alpha \mapsto-2 \pi \int_{c} \alpha
$$

It is clear that if $\mathbf{d}=\left(d_{j}\right)$ is a different collection of paths joining $P$ to $P_{j}$ then the elements $\mathcal{P}_{\mathbf{c}}(D)$ and $\mathcal{P}_{\mathbf{d}}(D)$ differ by an element in the lattice of periods $\Lambda$. We have thus defined a map

$$
\mathrm{AJ}: \operatorname{Div}_{0}(\Sigma) \rightarrow V / \Lambda, \quad D \mapsto \mathcal{P}(D)
$$


Abel-Jacobi Theorem The restriction of $\mathrm{AJ}$ to $\operatorname{PDiv}(\Sigma)$ is trivial and the induced map

$$
\mathrm{AJ}: \operatorname{Div}_{0}(\Sigma) / \operatorname{PDiv}(\Sigma) \rightarrow V / \Lambda
$$

is an isomorphism of abelian groups.

With the advent of sheaf theory, in the 1950's came Kodaira's observation of a bijection between the degree zero divisors and degree zero holomorphic line bundles over a Riemann surface. The associated line bundle is holomorphically trivial if and only if the divisor is principal (see [4]). Thus, we can identify $\operatorname{Div}_{0}(\Sigma) / \operatorname{PDiv}(\Sigma)$ with the abelian group $\operatorname{Pic}_{0}(\Sigma)$ of isomorphism classes of holomorphic line bundles of degree zero over $\Sigma$. (The group structure on $\mathrm{Pic}_{0}(\Sigma)$ is given by the tensor product of line bundles.) The Abel-Jacobi theorem describes a group isomorphism between $\operatorname{Pic}_{0}(\Sigma)$ and the Jacobian $J(\Sigma):=V / \Lambda$.

Our proof uses the correspondence between holomorphic vector bundles and connection mod gauge equivalence (see the influential [1]). A holomorphic structure on a line bundle can be defined in terms of a connection (and its associated Cauchy-Riemann operator). Two connections define the same holomorphic structure if they lie in the same orbit of the gauge group.

J. B. Bost has described in [2] one possible elementary gauge theoretic approach to the Abel-Jacobi theorem. The goal of the present note is to present a different, and in our view, more structured gauge theoretic approach to this beautiful classical result. We associate to each connection on the (topologically) trivial line bundle over $\Sigma$ an element in $J(\Sigma)$ which does not depend on the gauge orbit of the connection. This is a complete orbit invariant meaning that connections in different orbits have different invariants. According to the previous discussion, we have a bijection $\operatorname{Pic}_{0}(\Sigma) \rightarrow J(\Sigma)$. The classical Abel-Jacobi theorem will follow from a tricky use of the Poincaré-Lefschetz duality theorem.

This paper is divided into three sections. In Section 1 we present the description of $\operatorname{Pic}_{0}(\Sigma)$ as a quotient of a certain action of the gauge group. Section 2 identifies this quotient with a torus. This leads to a proof of the Abel-Jacobi theorem in Section 3.

\section{Holomorphic Structures and Connections on Line Bundles}

A line bundle over $\Sigma$ is given by an open cover $\left(U_{i}\right)$ of $\Sigma$ and a gluing cocycle

$$
g_{j i}: U_{i j}:=U_{i} \cap U_{j} \rightarrow \mathbb{C}^{*}
$$

A holomorphic structure is then defined by a choice of gluing cocycle such that all the maps $g_{i j}$ are holomorphic. For example, suppose we are given a divisor $D=\sum_{j=1}^{m} n_{j} p_{j} \in$ $\operatorname{Div}(\Sigma)$. Here the points $p_{j}$ are assumed pairwise distinct. Pick pairwise disjoint coordinate disks $U_{j}$ centered at $p_{j}$ and denote by $z_{j}$ a local holomorphic coordinate on $U_{j}$. Now define

$$
U_{0}=\Sigma \backslash\left\{p_{1}, \ldots, p_{m}\right\}
$$

The gluing cocycle

$$
g_{0 j}: U_{j} \cap U_{0} \rightarrow \mathbb{C}^{*}, \quad g_{0 j}\left(z_{j}\right)=z_{j}^{-n_{j}}
$$


defines a holomorphic line bundle $E(D)$, called the holomorphic line bundle associated to the divisor $D$. In [4] it is shown that this correspondence induces an isomorphism

$$
\operatorname{Div}_{0}(\Sigma) / \operatorname{PDiv}(\Sigma) \cong \operatorname{Pic}_{0}(\Sigma)
$$

We will denote by $\operatorname{Holo}(E)$ the space of holomorphic structures on a line bundle $E$ so that, if $\operatorname{deg} E=0$ we have an isomorphism

$$
\operatorname{Pic}_{0}(\Sigma) \cong \operatorname{Holo}(E) / \sim
$$

where the equivalence relation is given by the isomorphism of two holomorphic structures. Fix now, once and for all, the degree zero smooth complex line bundle $E \rightarrow \Sigma$.

There exists an alternate approach of defining a holomorphic structure on $E$ using the Cauchy-Riemann (CR) operators on $E$ (or partial connections in the terminology of [2]). A CR-operator is a C-linear first order partial differential operator $\Omega^{0,0}(E) \rightarrow \Omega^{0,1}(E)$ such that

$$
L(f u)=(\bar{\partial} f) u+f L u, \quad \forall f: \Sigma \rightarrow \mathbb{C}, u \in C^{\infty}(E) .
$$

(We need not impose the usual condition $L^{2}=0$ since there are no $(0,2)$ forms on $\Sigma$.) Denote by $\mathrm{CR}(E)$ the space of CR-operators on $E$ and by $\mathcal{G}^{c}$ the complex gauge group consisting of smooth maps $T: \Sigma \rightarrow \mathbb{C}^{*} . \mathcal{G}^{c}$ acts on $\mathrm{CR}(E)$ by conjugation $L \stackrel{T}{\mapsto} T L T^{-1}$. In [5], Chapter 1 it is shown that

$$
\operatorname{Holo}(E) \cong \mathrm{CR}(E) \text { and } \operatorname{Pic}_{0}(\Sigma) \cong \mathrm{CR}(E) / \mathcal{G}^{c}
$$

Now denote by $\mathcal{A}^{c}$ the affine space of smooth connections on $E$ compatible with the complex structure (only). An element of $\mathcal{A}^{c}$ is a linear first order partial differential operator $\nabla^{A}: \Omega^{0}(E) \rightarrow \Omega^{1}(E)$ satisfying the product rule

$$
\nabla^{A}(f u)=(d f) u+f \nabla^{A} u, \quad \forall f \in C^{\infty}(\Sigma), u \in \Omega^{0}(E) .
$$

$\mathcal{A}^{c}$ is an affine space modeled by $\Omega^{1}(\Sigma) \otimes \mathbb{C}$. To see this observe that the difference of two covariant derivatives commutes with the multiplication by a smooth function

$$
\left(\nabla^{A}-\nabla^{B}\right)(f u)=f\left(\nabla^{A}-\nabla^{B}\right) u
$$

If we denote the contraction by a vector field $X$, by $i_{X}$ and the composition $i_{X} \circ \nabla^{A}$, by $\nabla_{X}^{A}$ then the difference $\delta:=\left(\nabla^{A}-\nabla^{B}\right)$ is the 1 -form given by

$$
\delta(X) \cdot u=\nabla_{X}^{A} u-\nabla_{X}^{B} u, \quad \forall X \in \operatorname{Vect}(\Sigma), u \in C^{\infty}(E)
$$

Now fix a connection, $\nabla^{A_{0}}$ on $E$. Using this connection and the above correspondence, we can represent any other connection by $\nabla^{A_{0}}+i A$, for some complex 1 -form $A$. We will often refer to $A$ as the connection. Again, $\mathcal{G}^{c}$ acts on $\mathcal{A}^{c}$ by conjugation. To any connection 
$A \in \mathcal{A}^{c}$ one can associate a CR-operator $\bar{\partial}_{A}:=P^{0,1} \nabla^{A}$ where $P^{0,1}: \Omega^{1}(E) \rightarrow \Omega^{0,1}(E)$ is the natural projection defined by the splitting $\Omega^{1}(E)=\Omega^{1,0}(E) \oplus \Omega^{0,1}(E)$. The map

$$
\mathcal{A}^{c} \ni A \mapsto \bar{\partial}_{A} \in \mathrm{CR}(E)
$$

is a $\mathcal{G}^{\mathcal{c}}$-equivariant surjection.

Any hermitian metric $h$ on $E$ defines an affine subspace $\mathcal{A}_{h} \subset \mathcal{A}^{c}$ consisting of metric compatible connections. These are characterized by the condition

$$
X \cdot h(u, v)=h\left(\nabla_{X}^{A} u, v\right)+h\left(u, \nabla_{X}^{A} v\right), \quad \forall X \in \operatorname{Vect}(\Sigma), u, v \in C^{\infty}(E) .
$$

The above equality shows that the difference $\delta=\nabla^{B}-\nabla^{A}$ between two such connections must be a purely imaginary 1 -form since

$$
0=h(\delta(X) u, v)+h(u, \delta(X) v)=(\delta(X)+\overline{\delta(X)}) h(u, v), \quad \forall X, u, v .
$$

Thus $\mathcal{A}_{h}$ is an affine space modeled by $i \Omega^{1}(\Sigma)$

Chern's theorem describes a procedure to construct right inverses to the map $\mathcal{A}^{c} \rightarrow$ $\mathrm{CR}(E)$. More precisely, given a CR operator, $L$, on $E$ and a metric $h$ on $E$, Chern's theorem (see $[4,5])$ states that there exists a unique connection $A=A(h, L) \in \mathcal{A}^{c}$ which is compatible with $h$ such that $\bar{\partial}_{A}=L$.

Fix one such metric, $h$ and denote by Chern $_{h}$ the map

$$
\operatorname{Chern}_{h}: \mathrm{CR}(E) \rightarrow \mathcal{A}_{h}, \quad L \mapsto A(h, L)
$$

postulated by Chern's theorem. The map Chern $h$ is a bijection, so we can transport the action of $\mathcal{G}^{c}$ on $\mathrm{CR}(E)$ to an action on $\mathcal{A}_{h}$. We obtain the isomorphisms

$$
\mathcal{A}_{h} / \mathcal{G}^{c} \cong \mathrm{CR}(E) / \mathcal{G}^{c} \cong \operatorname{Pic}_{0}(\Sigma) .
$$

We want to describe the action of $\mathcal{G}^{c}$ on $\mathcal{A}_{h}$ explicitly.

Fix $A \in \mathcal{A}_{h}$ and $L \in \mathrm{CR}(E)$ such that $A=A(h, L)$ and let $T \in \mathcal{G}^{c}$. Then we can write

$$
T=\exp (f) g, \quad f: \Sigma \rightarrow \mathbb{R}, g: \Sigma \rightarrow U(1) .
$$

Set $A_{T}=T \cdot A=A\left(h, T L T^{-1}\right)$. Then $A_{T}$ is determined by the following two requirements.

(i) $A_{T}$ is compatible with $h$.

(ii) $\bar{\partial}_{A_{T}}:=T L T^{-1}=T \bar{\partial}_{A} T^{-1}=\bar{\partial}_{A}-\bar{\partial} f-(d g / g)^{0,1}$, where for any complex valued 1 -form $\alpha$ on $\Sigma$ we denoted by $\alpha^{0,1}$ its $(0,1)$-part.

The condition (i) can be rephrased by saying that $A_{T}=A+i \omega$ where $\omega \in \Omega^{1}(\Sigma)$ while (ii) can be rephrased as

$$
(i \omega)^{(0,1)}=-\bar{\partial} f-(d g / g)^{(0,1)} .
$$

To get a better grasp of (1.5) it is convenient to work in local holomorphic coordinates $z=x+i y$ on $\Sigma$. Write $\omega=a d x+b d y$ and $d g / g=i u d x+i v d y$ with $a, b, u, v: \Sigma \rightarrow \mathbb{R}$. Rewriting $\omega=\frac{a-i b}{2} d z+\frac{a+i b}{2} d \bar{z}$, shows that

$$
(i \omega)^{(0,1)}=i \frac{a+i b}{2} d \bar{z} .
$$


Similarly,

$$
(i d g / g)^{(0,1)}=i \frac{u+i v}{2} d \bar{z}
$$

Since $\bar{\partial} f=\frac{1}{2}\left(f_{x}+i f_{y}\right)$ and $* d f=-f_{y} d x+f_{x} d y$, the real and imaginary parts of (1.5) give $a=-f_{y}-u$ and $b=f_{x}-v$ or

$$
A_{T}-A=\omega=* d f+i d g / g
$$

The last equality describes the action of $T$ on $A$.

\section{Orbit Invariants}

In this section we define a complete set of invariants for the action of $\mathcal{G}^{c}$ on $\mathcal{A}_{h}$. To begin, fix a trivial metric and connection on the trivial line bundle over $\Sigma$ for the remainder of this section. Recall that $\operatorname{Harm}^{1}(\Sigma)=\{\alpha \mid d \alpha=* d \alpha=0\}$ is the space of harmonic 1-forms.

Let $\operatorname{Harm}^{1}(\Sigma, 2 \pi \mathbb{Z})$ denote the subspace $\left\{\alpha \mid \int_{\gamma} \alpha \in 2 \pi \mathbb{Z}, \forall \gamma \in H_{1}(\Sigma, \mathbb{Z})\right\}$.

Define a map $\tilde{J}: i \Omega^{1}(\Sigma) \rightarrow \operatorname{Harm}^{1}(\Sigma)^{*}$ by

$$
\langle\tilde{J}(i \omega), \alpha\rangle=\int_{\Sigma} \omega \wedge \alpha, \quad \forall i \omega \in i \Omega^{1}(\Sigma), \alpha \in \operatorname{Harm}^{1}(\Sigma)
$$

Here $\langle$ ?, ? $\rangle$ denotes the natural pairing between a vector space and its dual. Denote by $\Lambda_{0}$ the lattice $\tilde{J}\left(i \operatorname{Harm}^{1}(\Sigma, 2 \pi \mathbb{Z})\right)$ in $\operatorname{Harm}^{1}(\Sigma)^{*}$. The next proposition states that the map

$$
J: i \Omega^{1}(\Sigma) \rightarrow \operatorname{Jac}(\Sigma):=\operatorname{Harm}^{1}(\Sigma) / \Lambda_{0} .
$$

induces an isomorphism, $\operatorname{Pic}_{0}(\Sigma) \cong \mathcal{A}_{h} / \mathcal{G}^{c} \rightarrow \operatorname{Jac}(\Sigma)$. This is what we mean by a complete set of invariants for the action of $\mathcal{G}^{c}$ on $\mathcal{A}_{h}$.

Proposition 2.1 The above map is a surjection and the following conditions are equivalent for $\omega_{1}, \omega_{2} \in i \Omega^{1}(\Sigma)$

(a) $J\left(\omega_{1}\right)=J\left(\omega_{2}\right)$.

(b) There exists $T \in \mathcal{G}^{c}$ such that $\omega_{2}=T \cdot\left(\omega_{1}\right)$.

Proof of the Proposition To see that $\tilde{J}$ is surjective, notice that the formal adjoint of $\tilde{J}$ is given by $\tilde{J}^{*}(\omega)(\alpha)=\int_{\Sigma} \omega \wedge \alpha$. If $\omega \in \operatorname{Co} \operatorname{ker} \tilde{J}$, then $\tilde{J}^{*}(\omega)(\alpha)=0$ for every $\alpha$, thus $\|\omega\|^{2}=\tilde{J}^{*}(\omega)\left(\omega^{*}\right)=0$. To establish the equivalence of (a) and (b) start with the Hodge decomposition of $\omega_{2}-\omega_{1}$. Namely

$$
\omega_{2}-\omega_{1}=\gamma+d a+d^{*} b
$$


Now we have,

$$
\begin{aligned}
J\left(\omega_{2}\right)(\alpha)-J\left(\omega_{1}\right)(\alpha) & =\int_{\Sigma} \gamma \wedge \alpha+\int_{\Sigma}(d a) \wedge \alpha-\int_{\Sigma}\left(* d^{*} b\right) \wedge \alpha \\
& =\int_{\Sigma} \gamma \wedge \alpha+\int_{\Sigma} d(a \wedge \alpha)-\int_{\Sigma} a \wedge d \alpha-\int_{\Sigma} d^{*} b \wedge * \alpha \\
& =\int_{\Sigma} \gamma \wedge \alpha-\int_{\Sigma} d(* b \wedge * \alpha)+\int_{\Sigma} * b \wedge d^{*} \alpha=\int_{\Sigma} \gamma \wedge \alpha
\end{aligned}
$$

Thus, $J\left(\omega_{1}\right)=J\left(\omega_{2}\right)$ if and only if $\gamma \in H^{1}(\Sigma, 2 \pi \mathbb{Z})$. This occurs exactly when there exists a $g: \Sigma \rightarrow U(1)$ so that $i d g / g=\gamma+d a$. Let $f=-* b$, then $J\left(\omega_{1}\right)=J\left(\omega_{2}\right)$ if and only if $\omega_{2}=T \cdot\left(\omega_{1}\right)$ with $T=\exp (f) g$. This proves that $\tilde{J}$ induces a well-defined isomorphism.

The definition of the Jacobian given is this section differ slightly from the definition given in the introduction. In order to reconcile this difference, notice that $*$ induces a complex structure on $\operatorname{Harm}^{1}(\Sigma)$ since $*^{2}=-1$. Extend it by complex linearity to $\operatorname{Harm}^{1}(\Sigma) \otimes$ (C Hodge theory on $\Sigma$ identifies the - $i$-eigenspace with the complex space $H^{0}\left(K_{\Sigma}\right)$ of holomorphic 1-forms on $\Sigma$. A complex conjugate linear isomorphism of complex vector spaces

$$
\operatorname{Harm}^{1}(\Sigma) \rightarrow H^{0}\left(K_{\Sigma}\right)
$$

is given by $\alpha \mapsto \frac{1}{2}(\alpha+i * \alpha)$. Thus the period map is completely determined by the complex period map

$$
J^{c}: i \Omega^{1}(\Sigma) \rightarrow H^{0}\left(K_{\Sigma}\right)^{*} / \Lambda_{0}, J^{c}(i \omega): \beta \mapsto \int_{\Sigma} \omega \wedge \beta, \quad \beta \in H^{0}\left(K_{\Sigma}\right)
$$

Note that the lattice $\Lambda_{0}$ corresponds, via the Poincare duality, to the lattice $\Lambda$ from the introduction. We now only have to show that $\operatorname{Pic}_{0}(\Sigma) \cong \operatorname{Div}_{0}(\Sigma) / \operatorname{PDiv}(\Sigma)$.

The previous proposition fits into the general framework of geometric invariant theory and symplectic reduction. These theories are used to describe quotients of algebraic varieties or symplectic manifolds by Lie groups. If $G$ is a Lie group and $G^{c}$ is the complexification of $G$, then

$$
X / / G \cong X / G^{c} \cong \mu^{-1}(0) / G
$$

Here, $m u$ is a moment map. Proposition (2.1) may be proved as an infinite dimensional version of this situation. The relevant Lie Group for Proposition 2.1 is the subgroup of $G^{c}$ consisting of unitary transformations. The complexification is $\mathcal{G}^{c}$ and the moment map is the curvature $F$. The result is that the Jacobian may also be identified with the space of flat connections modulo unitary gauge transformations. These ideas have been exploited through gauge theory. See [1], [3, Chapter 6]. Perhaps the most famous example is Donaldson's identification of the moduli space of anti-self dual connections with the moduli of rank 2 stable vector bundles over algebraic surfaces. 


\section{The Abel-Jacobi Theorem}

In this section we will complete the proof of the Abel-Jacobi theorem by proving that Jacobian may also be identified with the group of degree zero divisors modulo principal divisors.

Let $D=\sum_{j=1}^{m} n_{j} P_{j} \in \operatorname{Div}_{0}(\Sigma)$. The associated holomorphic line bundle $E(D)$ can be described using the cover $U_{0}, U_{1}, \ldots, U_{m}$ described in (1.1) and the gluing cocycle (1.2). A hermitian metric $h$ on $E(D)$ is given by a collection of positive functions

$$
h_{j}: U_{j} \rightarrow(0, \infty), \quad j=0,1, \ldots, m
$$

satisfying the gluing rules $h_{0}=\left|g_{0 j}\right|^{-2} h_{j}$, on $U_{0} \cap U_{j}, j=1, \ldots, m$. The Chern connection $A$ associated to the pair $(E(D), h)$ is given by the collection of complex valued 1-forms (see $[4$, Chapter 1])

$$
\theta_{j}=\frac{\partial h_{j}}{h_{j}}, \quad j=0,1, \ldots, m
$$

which along the overlaps satisfy the transition rules

$$
\theta_{0}=\theta_{j}+n_{j} \frac{d z_{j}}{z_{j}}
$$

The bundle $E(D)$ is topologically trivial. Fix such a trivialization and denote by $\vec{s}$ the constant section 1 . In terms of the above gluing cocycle, the section $\vec{s}$ can be described as a collection of maps $s_{j}: U_{j} \rightarrow \mathbb{C}^{*}$ satisfying the gluing rules $s_{j}=g_{j 0} s_{0}$ or equivalently,

$$
g_{j 0}=s_{j} / s_{0} .
$$

Fix the metric $h$ so that $\vec{s}$ has constant pointwise norm 1 . We deduce that

$$
h_{j}=\left|s_{j}\right|^{-2}, \quad j=0,1, \ldots, m .
$$

Hence

$$
\theta_{j}=\left|s_{j}\right|^{2} \partial\left(\left|s_{j}\right|^{-2}\right)=-\frac{\bar{s}_{j} \partial s_{j}+s_{j} \partial \bar{s}_{j}}{s_{j} \bar{s}_{j}}=-\frac{\partial s_{j}}{s_{j}}-\frac{\partial \bar{s}_{j}}{\bar{s}_{j}} .
$$

Denote by $A_{0}$ the trivial connection (determined by the trivialization) and by $A$ the Chern connection determined by the gluing cocycle $g_{j 0}$ and the metric $h$. To prove the Abel-Jacobi theorem it suffices to show that

$$
\tilde{J}\left(A-A_{0}\right)=\mathcal{P}(D) \bmod \Lambda .
$$

To show this we need to know more about $A-A_{0}$.

Denote by $\nabla^{0}$ the covariant derivative determined by $A_{0}$. In terms of the gluing cocycle $g_{j 0}$, the connection $A_{0}$ is given by a collection of complex 1-forms $\beta_{j}$ satisfying

$$
\beta_{j}-\beta_{0}=-n_{j} \frac{d z_{j}}{z_{j}}
$$


on the overlaps. Moreover, the condition $\nabla^{0} \vec{s}=0$ implies that $d s_{j}+\beta_{j} s_{j}=0$ so that $\beta_{j}=-d s_{j} / s_{j}$. The difference $A_{0}-A$ is given by the collection of 1 -forms

$$
\delta_{j}:=\beta_{j}-\theta_{j}=-\frac{\bar{\partial} s_{j}}{s_{j}}+\frac{\partial \bar{s}_{j}}{\bar{s}_{j}} .
$$

The equalities (3.1) and (3.4) show that the forms $\delta_{j}$ agree on the overlaps and thus determine a global 1-form. Set $\omega:=A-A_{0}$, so that $\left.i \omega\right|_{U_{j}}=\delta_{j}$.

To compute $\tilde{J}^{c}(\omega)$ assume that the coordinate patches $U_{j}$ are biholomorphic to the unit disk in the plane. Let $U_{j}(r) \subset U_{j}$ denote the sub-disk of radius $r<1$ centered at the origin. Set

$$
\Sigma_{r}=\Sigma \backslash\left(\bigcup_{j=1}^{m} U_{j}(r)\right) .
$$

Given a holomorphic 1-form $\alpha$ on $\Sigma$,

$$
\left\langle\tilde{J}^{c}(\omega), \alpha\right\rangle=-i \lim _{r \rightarrow 0} \int_{\Sigma_{r}} \delta_{0} \wedge \alpha
$$

Observe that $\delta_{0} \wedge \alpha=-\frac{d s_{0}}{s_{0}} \wedge \alpha$ since $\alpha$ is a (1,0)-form. Furthermore, we may choose a function $\beta: \Sigma \rightarrow \mathbb{C}$ such that $\left.\beta\right|_{\Sigma_{r}}=0$ and $\alpha=d \beta$ on each $U_{j}$, by the Poincaré lemma. This gives:

$$
\begin{aligned}
\left\langle\tilde{J}^{c}(\omega), \alpha\right\rangle & =i \lim _{r \rightarrow 0} \int_{\Sigma_{r}} \frac{d s_{0}}{s_{0}} \wedge \alpha_{0}+i \lim _{r \rightarrow 0} \int_{\Sigma_{r}} \frac{d s_{0}}{s_{0}} \wedge d \beta \\
& =i \lim _{r \rightarrow 0} \int_{\Sigma_{r}} \frac{d s_{0}}{s_{0}} \wedge \alpha_{0}-i \sum_{j=1}^{m} \lim _{r \rightarrow 0} \int_{\partial U_{j}(r)} \beta \frac{d s_{0}}{s_{0}} \\
& =i \lim _{r \rightarrow 0} \int_{\Sigma_{r}} \frac{d s_{0}}{s_{0}} \wedge \alpha_{0}
\end{aligned}
$$

We used Stokes' theorem to get the second line.

The form $-\frac{i}{2 \pi} d s_{0} / s_{0}$ is closed and therefore represents an element of $H^{1}(\Sigma, \mathbb{C})$. In fact it represent an element of $H^{1}(\Sigma, \mathbb{Z})$. Therefore, by Lefschetz duality, there is a 1-cycle $c$ (represented as a formal linear combination of oriented 1-manifolds) with the property:

$$
-\frac{i}{2 \pi} \int_{\Sigma_{r}} \frac{d s_{0}}{s_{0}} \wedge \gamma=\int_{c} \gamma
$$

for any closed form $\gamma$ with compact support. Using the gluing formula, $s_{0}=z_{j}^{-n_{j}} s_{j}$, we may compute that

$$
-\frac{d s_{0}}{s_{0}}=n_{j} \frac{i}{2 \pi} \frac{d z_{j}}{z_{j}}-\frac{i}{2 \pi} \frac{d s_{j}}{s_{j}}
$$




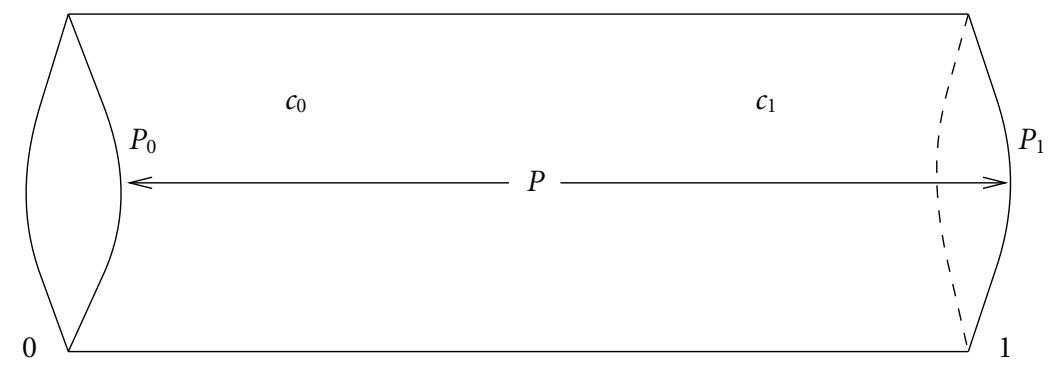

Figure 1: The Poincaré-Lefschetz dual of $\frac{1}{2 \pi} d \theta$ on a cylinder

on the overlap. Since $-\frac{i}{2 \pi} \frac{d z_{j}}{z_{j}}$ generate the integral cohomology of the boundary components and $-\frac{i}{2 \pi} \frac{d s_{j}}{s_{j}}$ is exact on $U_{j}$, we see that $-\frac{i}{2 \pi} \frac{d s_{0}}{s_{0}}$ is dual $\sum_{j=1}^{m} n_{j} c_{j}+c_{0}$. Here each $c_{j}$ is a smooth path in $\Sigma$, connecting a fixed point $p$ to $p_{j}$ and $c_{0}$ is a formal sum of closed curves. We may check our sign conventions with the example in Figure 1. Orient the boundary using the outward normal first convention (see [6]). The Lefschetz dual of $\frac{i}{2 \pi} d \theta$ will be given by $c_{0}-c_{1}$.

We can now complete the proof:

$$
\begin{aligned}
\left\langle\tilde{J}^{c}(\omega), \alpha\right\rangle & =i \lim _{r \rightarrow 0} \int_{\Sigma_{r}} \frac{d s_{0}}{s_{0}} \wedge \alpha_{0}=-2 \pi \sum_{j=1}^{m} \int_{c_{j}} \alpha_{0}=-2 \pi \sum_{j=1}^{m} n_{j} \int_{c_{j}}(\alpha-d \beta) \\
& =-2 \pi \sum_{j=1}^{m} n_{j} \int_{c_{j}} \alpha+2 \pi \sum_{j=1}^{m} n_{j} \beta(P)=-2 \pi \sum_{j=1}^{m} n_{j} \int_{c_{j}} \alpha=\mathcal{P}(D)(\alpha)
\end{aligned}
$$

This is the classical Abel-Jacobi theorem.

\title{
References
}

[1] M. F. Atiyah and R. Bott, The Yang-Mills equations over Riemann surfaces. Philos. Trans. Roy. Soc. London Ser. A 308(1982), 523-615.

[2] J. B. Bost, Introduction to Compact Riemann Surfaces, Jacobians and Abelian Varieties. In: From Number Theory to Physics, Springer Verlag.

[3] S. K. Donaldson and P. B. Kronheimer, The Geometry of Four-Manifolds. Oxford Science Publications, 1990.

[4] P. Griffiths and J. Harris, Principles of Algebraic Geometry. John Wiley \& Sons, 1978.

[5] S. Kobayashi, Differential Geometry of Complex Vector Bundles. Princeton University Press, 1987.

[6] L. I. Nicolaescu, Lectures on the Geometry of Manifolds. World Scientific, 1996.

\author{
Seminarul Matematic \\ Al.Myller University \\ Al.I. Cuza, Iaşi \\ 6600 Romania \\ e-mail:giones@uaic.ro
}

